PROCEEDINGS OF THE

AMERICAN MATHEMATICAL SOCIETY

Volume 131, Number 2, Pages 415-423

S 0002-9939(02)06665-0

Article electronically published on September 17, 2002

\title{
NONEXISTENCE RESULTS FOR HIGHER-ORDER EVOLUTION PARTIAL DIFFERENTIAL INEQUALITIES
}

\author{
GENNADY G. LAPTEV
}

(Communicated by David S. Tartakoff)

\begin{abstract}
Nonexistence of global solutions to semilinear higher-order (with respect to $t$ ) evolution partial differential inequalities $u_{t}^{(k)}-\Delta u \geq|x|^{\sigma}|u|^{q}$ with $k=1,2, \ldots$ in the complement of a ball is studied. The critical exponents $q^{*}$ are found and the nonexistence results are proved for $1<q \leq q^{*}$. The corresponding results for $k=1$ (parabolic problem) are sharp.
\end{abstract}

\section{INTRODUCTION}

In this paper we study the nonexistence of global solutions for higher-order (with respect to $t$ ) semilinear partial differential inequalities

$$
\frac{\partial^{k} u}{\partial t^{k}}-\Delta u \geq|x|^{\sigma}|u|^{q}, \quad(x, t) \in \Omega \times(0, \infty) .
$$

Let us introduce the "critical singularity" $\sigma^{*}=-2$. We consider the problem in the complement of a ball $\Omega=\mathbf{R}^{N} \backslash B_{R}, R>0$, and in the case $+\infty>\sigma>\sigma^{*}$ we obtain the critical exponent $q^{*}$. In another case, $-\infty<\sigma \leq \sigma^{*}$, for the problem in the ball $\Omega=B_{R}$ we formulate some results from the author's paper [17] (see also [6, 29]) on nonexistence for all $q>1$.

Corresponding investigations for parabolic equations were initiated by Bandle and Levine [2] (see also the references therein).

Nonexistence theory for evolution equations is well developed. We do not cite all the results, but let us mention the book [30] and the surveys [20, 8] (parabolic problems), and the books [1, 12, 24] and the papers [7, 10] (hyperbolic problems).

In this paper we obtain some generalization for higher-order evolution equations and inequalities, which includes, among others, parabolic and hyperbolic problems. We use the nonlinear test-function method, developed by Pohozaev [27], Mitidieri and Pohozaev [22, 23, 24, Pohozaev and Tesei [28, Veron and Pohozaev [32, and Zhang [33, 34, 35] (see also the papers by Bandle, Levine and Zhang [3], Kurta [14, 15, Levine and Zhang [21] and author's papers [16, 17, 18, 19]).

Let $N \geq 3, Q$ be an (unbounded) domain in $\mathbf{R}^{N+1}$ with piecewise smooth boundary. We will use the well-known Sobolev spaces $W_{q}^{2}(Q)$ and the local space $L_{q, \text { loc }}(Q)$, the elements of which belong to $L_{q}\left(Q^{\prime}\right)$ for any compact subset $Q^{\prime}: \overline{Q^{\prime}} \subset$

Received by the editors June 10, 2001.

2000 Mathematics Subject Classification. Primary 35G25; Secondary 35R45, 35K55, 35L70.

The author was supported in part by INTAS project 00-0136 and RFBR Grant \#01-01-00884.

(C)2002 American Mathematical Society 
Q. Denote the space of continuous functions by $C(\bar{Q})$ and the space of smooth functions by $C^{m}(\bar{Q})$. Similar anisotropic spaces $W_{q}^{2, k}(Q)$ and $C^{2, k}(\bar{Q})$ are also introduced.

The symbol $\Delta$ stands for the Laplace operator. The expression $\frac{\partial u}{\partial n}$ denotes the derivative of $u$ in the direction of the outward normal $n$ to the boundary of the domain under consideration. Indexed $c$ 's and $C$ 's are used to designate constants.

\section{MAin Results}

Let $R>0, k \in \mathbf{N}$ and $-2<\sigma<+\infty$. Let us introduce the domain $\Omega=\mathbf{R}^{N} \backslash B_{R}$ and consider the problem

$$
\begin{cases}\frac{\partial^{k} u}{\partial t^{k}}-\Delta u \geq|x|^{\sigma}|u|^{q}, & (x, t) \in \Omega \times(0, \infty), \\ u(x, t) \geq 0, & (x, t) \in \partial \Omega \times(0, \infty), \\ \frac{\partial^{k-1} u}{\partial t^{k-1}}(x, 0) \geq 0, & x \in \Omega .\end{cases}
$$

Definition 1.1. Let $u(x, t) \in C(\bar{\Omega} \times[0, \infty))$ and the locally integrable traces $\frac{\partial^{i} u}{\partial t^{i}}(x, 0), i=1, \ldots, k-1$, are well defined. The function $u(x, t)$ is called a weak solution to problem (1.1) if, for any nonnegative test-function $\varphi(x, t) \in W_{\infty}^{2, k}(\Omega \times(0, \infty))$ with compact support, such that $\left.\varphi\right|_{\partial \Omega \times(0, \infty)}=0$, the integral inequality

$$
\begin{gathered}
\int_{0}^{\infty} \int_{\partial \Omega} u \frac{\partial \varphi}{\partial n} d x d t+\int_{0}^{\infty} \int_{\Omega} u\left((-1)^{k} \frac{\partial^{k} \varphi}{\partial t^{k}}-\Delta \varphi\right) d x d t \\
\geq \int_{0}^{\infty} \int_{\Omega}|x|^{\sigma}|u|^{q} \varphi d x d t+\sum_{i=1}^{k-1}(-1)^{i} \int_{\Omega} \frac{\partial^{k-1-i} u}{\partial t^{k-1-i}}(x, 0) \frac{\partial^{i} \varphi}{\partial t^{i}}(x, 0) d x \\
\quad+\int_{\Omega} \frac{\partial^{k-1} u}{\partial t^{k-1}}(x, 0) \varphi(x, 0) d x
\end{gathered}
$$

holds.

Theorem 1.2. For $\sigma>-2$ and

$$
1<q \leq q_{k}^{*}=\frac{N+2 / k+\sigma}{N-2+2 / k}=1+\frac{2+\sigma}{N-2+2 / k}
$$

problem (1.1) has no nontrivial global weak solution.

This theorem includes, among others, the sharp results for parabolic problems $\left(k=1\right.$, Fujita-Hayakawa's critical exponent $q_{1}^{*}=1+\frac{2+\sigma}{N}$; see also [25, 4, 26, 20, 25. $8,3,21])$ and hyperbolic problems ( $k=2$, Kato's critical exponent $q_{2}^{*}=1+\frac{2+\sigma}{N-1}$; see also [32, 34, 9]). It is interesting that formally passing to the limit as $k \rightarrow \infty$ in Theorem 1.2 we arrive at the sharp elliptic critical exponent $q_{\infty}^{*}=\frac{N+\sigma}{N-2}$ (see, for example, 22, 23, 24, 13, 16, 5] and the references therein).

Now let $-\infty<\sigma \leq-2$. Let us consider the problem (1.1) in the ball $B_{R}$, i.e. $\Omega=B_{R}$.

Definition 1.3. Let $u(x, t) \in C(\bar{\Omega} \backslash\{0\} \times[0, \infty))$ and let the traces $\frac{\partial^{i} u}{\partial t^{i}}(x, 0) \in$ $L_{\mathrm{loc}}(\Omega \backslash\{0\}), i=1, \ldots, k-1$, be well defined. The function $u(x, t)$ is called a weak solution to problem (1.1) if, for any nonnegative test-function $\varphi(x, t) \in$ $W_{\infty}^{2, k}(\Omega \times(0, \infty))$, such that $\varphi(x, t) \equiv 0$ in a neighborhood of $x=0$ for all $t \geq 0$ and $\left.\varphi\right|_{\partial \Omega \times(0, \infty)}=0$, the integral inequality (1.2) holds. 
Theorem 1.4 ([17]). For $-\infty<\sigma \leq-2$ and

$$
1<q<+\infty
$$

problem (1.1) in the ball $\Omega=B_{R}$ has no global (with respect to t) nontrivial solution.

Let us explain the meaning of the above theorem. To do this, it is convenient to use an analogy with Theorem 1.2 In the case $\sigma \leq-2$, the solution has a singularity at $x=0$ rather than at infinity. Accordingly, the test function vanishes in a neighborhood of $x=0$, and no assumption is made about the integrability of $u(x, t)$ at zero. This argument can be formalized by applying the Kelvin transform in order to proceed to an exterior problem in $\mathbf{R}^{N} \backslash B_{R}$.

\section{Auxiliary estimates}

In this section we obtain some estimates depending on the parameter $\rho, \rho \rightarrow \infty$. These estimates play a fundamental role in the test function method.

Let us consider the "standard cut-off function" $\zeta(y) \in C^{\infty}\left(\mathbf{R}_{+}\right)$with the following properties:

$$
0 \leq \zeta(y) \leq 1, \quad \zeta(y)= \begin{cases}1, & \text { if } 0 \leq y \leq 1, \\ 0, & \text { if } y \geq 2\end{cases}
$$

For the function

$$
\eta(y)=(\zeta(y))^{k p_{0}}
$$

with some positive $p_{0}$ and $k \in \mathbf{N}$, by direct calculation one can obtain the estimates (for $1<p \leq p_{0}$ )

$$
\begin{aligned}
& \left|\eta^{\prime}(y)\right|^{p}=\left(k p_{0}\right)^{p} \zeta^{k p_{0}(p-1)} \zeta^{k p_{0}-p}\left|\zeta^{\prime}\right|^{p} \leq c_{\eta} \eta^{p-1}(y), \\
& \left|\eta^{\prime \prime}(y)\right|^{p} \leq\left(k p_{0}\right)^{p} \zeta^{k p_{0}(p-1)} \zeta^{k p_{0}-2 p}\left(\left(k p_{0}-1\right)\left|\zeta^{\prime}\right|^{2}+\zeta\left|\zeta^{\prime \prime}\right|\right)^{p} \leq c_{\eta} \eta^{p-1}(y), \\
& \quad \ldots \\
& \left|\eta^{(k)}(y)\right|^{p} \leq c_{\eta} \eta^{p-1}(y)
\end{aligned}
$$

with a positive constant $c_{\eta}$.

Now let us introduce the change of variables $y=t / \rho^{\theta}$, with $\theta>0, \rho>2 R$. For the function $\eta\left(t / \rho^{\theta}\right)$ we have

$$
\operatorname{supp}\left|\eta\left(\frac{t}{\rho^{\theta}}\right)\right|=\left\{t<2 \rho^{\theta}\right\}, \quad \operatorname{supp}\left|\frac{d^{k} \eta\left(t / \rho^{\theta}\right)}{d t^{k}}\right|=\left\{\rho^{\theta}<t<2 \rho^{\theta}\right\},
$$

and

$$
\int_{\text {supp }\left|\frac{d^{k} \eta\left(t / \rho^{\theta}\right)}{d t^{k}}\right|} \frac{\left|\frac{d^{k} \eta\left(t / \rho^{\theta}\right)}{d t^{k}}\right|^{p}}{\eta^{p-1}\left(t / \rho^{\theta}\right)} d t \leq c_{\eta} \rho^{-\theta(k p-1)} .
$$

The parameter $\theta$ will be chosen later.

For the variable $x,|x|=r$, we introduce the functions $\eta(r / \rho)$,

$$
\xi(x) \equiv \xi(r)=\frac{1}{R^{s}}-\frac{1}{r^{s}},
$$

and

$$
\psi_{\rho}(x) \equiv \psi_{\rho}(r)=\left(\frac{1}{R^{s}}-\frac{1}{r^{s}}\right) \eta\left(\frac{r}{\rho}\right) .
$$

Here we will put $s=N-2$. It is evident that $\psi_{\rho}=0$ and $\frac{\partial \psi_{\rho}}{\partial r} \geq 0$ as $r=R$. 
For the derivatives of the function $\psi_{\rho}(r)$ (as $\left.r>2 R\right)$ we have

$$
\begin{gathered}
\left|\frac{\partial \psi_{\rho}}{\partial r}\right|^{p} \leq\left|\frac{s}{r^{s+1}} \eta\left(\frac{r}{\rho}\right)+\left(\frac{1}{R^{s}}-\frac{1}{r^{s}}\right) \eta^{\prime}\left(\frac{r}{\rho}\right) \frac{1}{\rho}\right|^{p} \leq c \eta^{p-1}\left(\frac{r}{\rho}\right) \frac{1}{R^{p s} r^{p}}\left(1+\frac{r^{p}}{\rho^{p}}\right) \\
\left|\frac{\partial^{2} \psi_{\rho}}{\partial r^{2}}\right|^{p} \leq\left|-\frac{s(s+1)}{r^{s+2}} \eta\left(\frac{r}{\rho}\right)+\frac{2 s}{r^{s+1} \rho} \eta^{\prime}\left(\frac{r}{\rho}\right)+\left(\frac{1}{R^{s}}-\frac{1}{r^{s}}\right) \frac{1}{\rho^{2}} \eta^{\prime \prime}\left(\frac{r}{\rho}\right)\right|^{p} \\
\leq c \eta^{p-1}\left(\frac{r}{\rho}\right) \frac{1}{R^{s p} r^{2 p}}\left(1+\frac{r^{p}}{\rho^{p}}+\frac{r^{2 p}}{\rho^{2 p}}\right)
\end{gathered}
$$

here $c$ does not depend on $r$ and $\rho$. Using these estimates we arrive at the inequality for the Laplace operator:

$$
\begin{aligned}
& \left|\Delta \psi_{\rho}(x)\right|^{p}=\left|\frac{\partial^{2} \psi_{\rho}}{\partial r^{2}}+\frac{N-1}{r} \frac{\partial \psi_{\rho}}{\partial r}\right|^{p} \leq c\left|\frac{\partial^{2} \psi_{\rho}}{\partial r^{2}}\right|^{p}+c \frac{1}{r^{p}}\left|\frac{\partial \psi_{\rho}}{\partial r}\right|^{p} \\
& \leq c \eta^{p-1}\left(\frac{r}{\rho}\right) \frac{1}{R^{s p} r^{2 p}}\left(1+\frac{r^{p}}{\rho^{p}}+\frac{r^{2 p}}{\rho^{2 p}}\right) \leq c \psi_{\rho}^{p-1}(x) \frac{1}{r^{2 p}}\left(1+\frac{r^{p}}{\rho^{p}}+\frac{r^{2 p}}{\rho^{2 p}}\right) .
\end{aligned}
$$

Now we take $s=N-2$. Due to

$$
\Delta\left(\frac{1}{r^{N-2}}\right)=0, \quad r \neq 0,
$$

we have $\Delta \psi_{\rho}=0$ for $r<\rho$ and

$$
\operatorname{supp}\left|\Delta \psi_{\rho}\right|=\{\rho<r<2 \rho\}
$$

On the set $\operatorname{supp}\left|\Delta \psi_{\rho}\right|$ the estimate

$$
1+\frac{r^{p}}{\rho^{p}}+\frac{r^{2 p}}{\rho^{2 p}} \leq c
$$

holds, where $c$ does not depend on $r$ and $\rho$. Therefore, it follows from (2.4) (for $\rho<r<2 \rho)$ that

$$
\left|\Delta \psi_{\rho}(x)\right|^{p} \leq c \psi_{\rho}^{p-1}(x) \frac{1}{\rho^{2 p}}
$$

hence for $\sigma \in \mathbf{R}$ we get

$$
\begin{aligned}
& \int_{\operatorname{supp}\left|\Delta \psi_{\rho}\right|} \frac{\left|\Delta \psi_{\rho}(x)\right|^{p}}{\psi_{\rho}^{p-1}(x)|x|^{\sigma(p-1)}} d x \\
& \quad \leq c \int_{\rho}^{2 \rho} \frac{\psi_{\rho}^{p-1}(x)}{\psi_{\rho}^{p-1}(x)} \frac{r^{N-1}}{\rho^{2 p+\sigma(p-1)}} d r \leq c_{\psi} \rho^{-p(\sigma+2)+N+\sigma} .
\end{aligned}
$$

Finally, for the general test-function

$$
\varphi_{\rho}(x, t)=\eta\left(\frac{t}{\rho^{\theta}}\right) \psi_{\rho}(x)
$$


we obtain the inequality

$$
\begin{aligned}
& \iint_{\operatorname{supp}\left|\Delta \varphi_{\rho}\right|} \frac{\left|\Delta \varphi_{\rho}(x, t)\right|^{p}}{\varphi_{\rho}^{p-1}(x, t)|x|^{\sigma(p-1)}} d x d t \\
& \quad \leq \int_{0}^{2 \rho^{\theta}} \eta\left(t / \rho^{\theta}\right) d t \int_{\operatorname{supp}\left|\Delta \psi_{\rho}\right|} \frac{\left|\Delta \psi_{\rho}\right|^{p}}{\psi_{\rho}^{p-1}|x|^{\sigma(p-1)}} d x \leq c_{\varphi} \rho^{\theta-p(\sigma+2)+N+\sigma} .
\end{aligned}
$$

Analogously, using (2.1), we obtain

$$
\begin{aligned}
& \text { (2.8) } \iint_{\operatorname{supp}\left|\frac{\partial^{k} \varphi_{\rho}}{\partial t^{k}}\right|}\left|\frac{\partial^{k} \varphi_{\rho}(x, t)}{\partial t^{k}}\right|^{p} \frac{1}{\varphi_{\rho}^{p-1}(x, t)|x|^{\sigma(p-1)}} d x d t \\
& \leq \int_{\operatorname{supp}\left|\frac{d^{k} \eta\left(t / \rho^{\theta}\right)}{d t^{k}}\right|}\left|\frac{d^{k} \eta\left(t / \rho^{\theta}\right)}{d t^{k}}\right|^{p} \frac{1}{\eta^{p-1}\left(t / \rho^{\theta}\right)} d t \int_{R<|x|<2 \rho} \frac{\psi_{\rho}(x)}{|x|^{\sigma(p-1)}} d x \\
& \leq c_{\eta} \rho^{-\theta(k p-1)} c \int_{R}^{2 \rho} \frac{r^{N-1}}{r^{\sigma(p-1)}} d r \leq c_{\varphi} \begin{cases}\rho^{N-\sigma(p-1)-\theta(k p-1)}, & N-\sigma(p-1)>0, \\
\rho^{-\theta(k p-1)} \ln \rho, & N-\sigma(p-1)=0, \\
\rho^{-\theta(k p-1)} R^{N-\sigma(p-1)}, & N-\sigma(p-1)<0 .\end{cases}
\end{aligned}
$$

For $\theta=2 / k$ the powers in these estimates (under condition $N-\sigma(p-1)>0$ ) are equal:

$$
N-\sigma(p-1)-\theta(k p-1)=\theta-p(\sigma+2)+N+\sigma \equiv-p(\sigma+2)+N+\sigma+2 / k .
$$

\section{Proof of Theorem 1.2}

Let $u(x, t)$ be a global nontrivial solution of problem (1.1). From Definition 1.1 with the test function $\varphi(x, t)=\varphi_{\rho}(x, t)$, defined by (2.6) with $p=q^{\prime}>1$ and $\theta=2 / k$, using the equalities

$$
\frac{\partial^{i} \varphi_{\rho}}{\partial t^{i}}(x, 0) \equiv 0, \quad i=1, \ldots, k-1,
$$

we obtain

$$
\begin{aligned}
& \int_{\Omega} \frac{\partial^{k-1} u}{\partial t^{k-1}}(x, 0) \varphi_{\rho}(x, 0) d x+\int_{0}^{\infty} \int_{\Omega}|u|^{q}|x|^{\sigma} \varphi_{\rho} d x d t \\
& \leq-\int_{0}^{\infty} \int_{\partial B_{R}} u \frac{\partial \varphi_{\rho}}{\partial r} d x d t+(-1)^{k} \iint_{\frac{\partial^{k} \varphi_{\rho}}{\partial t^{k}}=0} u \frac{\partial^{k} \varphi_{\rho}}{\partial t^{k}} d x d t-\iint_{\Delta \varphi_{\rho}=0} u \Delta \varphi_{\rho} d x d t \\
& +(-1)^{k} \iint_{\frac{\partial^{k} \varphi_{\rho}}{\partial t^{k}} \neq 0} u \frac{\partial^{k} \varphi_{\rho}}{\partial t^{k}} d x d t-\iint_{\Delta \varphi_{\rho} \neq 0} u \Delta \varphi_{\rho} d x d t .
\end{aligned}
$$

As it was mentioned above $\left.\frac{\partial \psi_{\rho}}{\partial r}\right|_{r=R} \geq 0$, so that $\left.\frac{\partial \varphi_{\rho}}{\partial r}\right|_{r=R} \geq 0$ and the first integral on the right-hand side is nonpositive due to our assumption $\left.u\right|_{\partial \Omega \times(0, \infty)} \geq 0$. The second and the third integrals equal zero. 
Using the Hölder inequality we obtain

$$
\begin{gathered}
\int_{\Omega} \frac{\partial^{k-1} u}{\partial t^{k-1}}(x, 0) \varphi_{\rho}(x, 0) d x+\int_{0}^{\infty} \int_{\Omega}|u|^{q}|x|^{\sigma} \varphi_{\rho} d x d t \\
=\int_{\Omega} \frac{\partial^{k-1} u}{\partial t^{k-1}}(x, 0) \varphi_{\rho}(x, 0) d x+\iint_{\varphi_{\rho}(x, t) \neq \xi(x)}|u|^{q}|x|^{\sigma} \varphi_{\rho} d x d t \\
\quad+\iint_{\varphi_{\rho}(x, t)=\xi(x)}|u|^{q}|x|^{\sigma} \xi(x) d x d t \\
\leq \iint_{\operatorname{supp}\left|\frac{\partial^{k} \varphi_{\rho}}{\partial t^{k}}\right|}|u|\left|\frac{\partial^{k} \varphi_{\rho}}{\partial t^{k}}\right| d x d t+\iint_{\operatorname{supp}\left|\Delta \varphi_{\rho}\right|}|u|\left|\Delta \varphi_{\rho}\right| d x d t
\end{gathered}
$$

$$
\begin{aligned}
& \leq\left(\iint_{\operatorname{supp}\left|\frac{\partial^{k} \varphi_{\rho}}{\partial t^{k}}\right|}|u|^{q}|x|^{\sigma} \varphi_{\rho} d x d t\right)^{1 / q}\left(\iint_{\operatorname{supp}\left|\frac{\partial^{k} \varphi_{\rho}}{\partial t^{k}}\right|} \frac{\left|\frac{\partial^{k} \varphi_{\rho}}{\partial t^{k}}\right|^{q^{\prime}}}{\varphi_{\rho}^{q^{\prime}-1}|x|^{\sigma\left(q^{\prime}-1\right)}} d x d t\right)^{1 / q^{\prime}} \\
& +\left(\iint_{\operatorname{supp}\left|\Delta \varphi_{\rho}\right|}|u|^{q}|x|^{\sigma} \varphi_{\rho} d x d t\right)^{1 / q}\left(\iint_{\operatorname{supp}\left|\Delta \varphi_{\rho}\right|} \frac{\left|\Delta \varphi_{\rho}\right|^{q^{\prime}}}{\varphi_{\rho}^{q^{\prime}-1}|x|^{\sigma\left(q^{\prime}-1\right)}} d x d t\right)^{1 / q^{\prime}}
\end{aligned}
$$

Finally, using the Young inequality, we get

$$
\begin{aligned}
\iint_{\varphi_{\rho}(x, t)=\xi(x)}|u|^{q}|x|^{\sigma} \xi(x) d x d t \leq c & \iint_{\operatorname{supp}\left|\frac{\partial^{k} \varphi_{\rho}}{\partial t^{k}}\right|} \frac{\left|\frac{\partial^{k} \varphi_{\rho}}{\partial t^{k}}\right|^{q^{\prime}}}{\varphi_{\rho}^{q^{\prime}-1}|x|^{\sigma\left(q^{\prime}-1\right)}} d x d t \\
& +c \iint_{\operatorname{supp}\left|\Delta \varphi_{\rho}\right|} \frac{\left|\Delta \varphi_{\rho}\right|^{q^{\prime}}}{\varphi_{\rho}^{q^{\prime}-1}|x|^{\sigma\left(q^{\prime}-1\right)}} d x d t .
\end{aligned}
$$

The last term due to (2.7) $\left(p=q^{\prime}, \theta=2 / k\right)$ is less than

$$
c \rho^{-q^{\prime}(\sigma+2)+N+\sigma+2 / k} \text {. }
$$

If

$$
-q^{\prime}(\sigma+2)+N+\sigma+2 / k \leq 0,
$$

then the last integral in $(3.3)$ is bounded as $\rho \rightarrow \infty$. As for the first integral in the right-hand side of (3.3), it is easy to see that under condition (3.4) this integral is bounded. Passing to the limit as $\rho \rightarrow \infty$ in the case (3.4) we obtain

$$
\int_{0}^{\infty} \int_{\Omega}|u|^{q}|x|^{\sigma} \xi d x d t \leq c_{0} .
$$

Then by the inequality $\varphi_{\rho} \leq \xi$ and taking into account that the Lebesgue integral is absolutely continuous with respect to the Lebesgue measure, we have

$$
\begin{gathered}
\iint_{\operatorname{supp}\left|\frac{\partial^{k} \varphi_{\rho}}{\partial t^{k}}\right|}|u|^{q}|x|^{\sigma} \varphi_{\rho} d x d t \leq \iint_{\operatorname{supp}\left|\frac{\partial^{k} \varphi_{\rho}}{\partial t^{k}}\right|}|u|^{q}|x|^{\sigma} \xi d x d t<\varepsilon(\rho) \rightarrow 0, \\
\iint_{\operatorname{supp}\left|\Delta \varphi_{\rho}\right|}|u|^{q}|x|^{\sigma} \varphi_{\rho} d x d t \leq \iint_{\operatorname{supp}\left|\Delta \varphi_{\rho}\right|}|u|^{q}|x|^{\sigma} \xi d x d t<\varepsilon(\rho) \rightarrow 0
\end{gathered}
$$

as $\rho \rightarrow \infty$. 
Then from inequality (3.2) we finally get

$$
\iint_{\varphi_{\rho}(x, t)=\xi(x)}|u|^{q}|x|^{\sigma} \xi(x) d x d t \leq 2 \varepsilon^{1 / q}(\rho) c_{0}^{1 / q^{\prime}} \rightarrow 0
$$

as $\rho \rightarrow \infty$, and

$$
\int_{0}^{\infty} \int_{\Omega}|u|^{q}|x|^{\sigma} \xi d x d t=0
$$

that is, the solution $u(x, t)$ must be trivial under condition (3.4), which is equivalent to the condition of Theorem 1.2

\section{Possible generalizations}

Using the described technique one can obtain nonexistence results for more general quasilinear equations and systems. Let us mention some examples in this direction.

For the inequality with damping

$$
\begin{cases}\frac{\partial^{k_{1}} u}{\partial t^{k_{1}}}+\frac{\partial^{k_{2}} u}{\partial t^{k_{2}}}-\Delta u \geq|x|^{\sigma}|u|^{q}, & (x, t) \in \Omega \times(0, \infty), \\ u(x, t) \geq 0, & (x, t) \in \partial \Omega \times(0, \infty), \\ \frac{\partial^{k_{1}-1} u}{\partial t^{k_{1}-1}}(x, 0) \geq 0, \frac{\partial^{k_{2}-1} u}{\partial t^{k_{2}-1}}(x, 0) \geq 0, & x \in \Omega,\end{cases}
$$

where $k_{1} \geq 1, k_{2} \geq 1$, we have

Theorem 4.1. Let $\Omega=\mathbf{R}^{N} \backslash B_{R},-2<\sigma<+\infty, k=\min \left\{k_{1}, k_{2}\right\}$ and $1<q \leq q_{k}^{*}$, where $q_{k}^{*}$ is defined in (1.3). Then problem (4.1) has no nontrivial global solution.

The special case of this problem $\left(k_{1}=1, k_{2}=2, \sigma=0\right)$ was investigated in [31] (on $\mathbf{R}^{N}$ ) and in [35] (on some noncompact manifolds) with additional assumption that initial data are compactly supported.

Lemma 4.2. Let $\Omega=\mathbf{R}^{N} \backslash B_{R}$ and

$$
1 \leq m<q \leq q^{*}=m+2 \frac{m-(m-1) / k}{N-2+2 / k} .
$$

Then the problem

$$
\begin{cases}\frac{\partial^{k} u}{\partial t^{k}}-\Delta u^{m} \geq|u|^{q}, & (x, t) \in \Omega \times(0, \infty), \\ u(x, t) \geq 0, & (x, t) \in \partial \Omega \times(0, \infty), \\ \frac{\partial^{k-1} u}{\partial t^{k-1}}(x, 0) \geq 0, & x \in \Omega,\end{cases}
$$

has no nontrivial global solution.

Lemma 4.3. Let $\Omega=\mathbf{R}^{N} \backslash B_{R}, q_{1}>1, q_{2}>1$, and

$$
\max \left\{\gamma_{1}, \gamma_{2}\right\} \geq \frac{N-2+2 / k}{2}, \quad \gamma_{1}=\frac{q_{1}+1}{q_{1} q_{2}-1}, \quad \gamma_{2}=\frac{q_{2}+1}{q_{1} q_{2}-1} .
$$

Then the problem

$$
\begin{cases}\frac{\partial^{k} u}{\partial t^{k}}-\Delta u \geq|v|^{q_{1}}, & (x, t) \in \Omega \times(0, \infty), \\ \frac{\partial^{k} v}{\partial t^{k}}-\Delta v \geq|u|^{q_{2}}, & (x, t) \in \Omega \times(0, \infty), \\ u(x, t) \geq 0, \quad v(x, t) \geq 0, & (x, t) \in \partial \Omega \times(0, \infty), \\ \frac{\partial^{k-1} u}{\partial t^{k-1}}(x, 0) \geq 0, \quad \frac{\partial^{k-1} v}{\partial t^{k-1}}(x, 0) \geq 0, & x \in \Omega\end{cases}
$$

has no nontrivial global solution. 
Remark 4.4. The referee's report on the earlier version of this paper contained a very useful remark that the method works if the complement of a circular cylinder (with respect to $t$ ) is replaced by the complement of other cylinder. In this case $R=R(t)$ and we have to assume that $R(t) \geq R_{0}>0$ (so the domain under consideration is infinite with respect to time $t$ ).

Finally it should be mentioned that the nonlinear test function method can be applied also to semilinear inequalities in cone-like domains (see, for example, the author's papers [16, 18, 19]).

\section{ACKNOWLEDGMENT}

The author is grateful to Professor H. Levine for bibliographic and historic remarks, Professor S. Pohozaev for setting up the problem and helpful discussion of the results, and the anonymous referee for useful comments which led to an improvement of the presentation of the results.

\section{REFERENCES}

[1] S. Alinhac, Blowup for nonlinear hyperbolic equations, Birkhäuser, Boston, 1995. MR 96h:35109

[2] C. Bandle and H.A. Levine, Fujita type results for convective-like reaction-diffusion equations in exterior domains, Z. Angew. Math. Phys. 40 (1989), 665-676. MR 90m:35093

[3] C. Bandle, H.A. Levine and Q. Zhang, Critical exponents of Fujita type for inhomogeneous parabolic equations and systems, J. Math. Anal. Appl. 251 (2000), 624-648. MR 2001h:35090

[4] P. Baras and R. Kersner, Local and global solvability of a class of semilinear parabolic equations, J. Differential Equations 68 (1987), 238-252. MR 88k:35089

[5] M.F. Bidaut-Veron and S.I. Pohozaev, Nonexistence results and estimates for some nonlinear elliptic problems, J. Anal. Math. 84 (2001), 1-49.

[6] H. Brezis and X. Cabré, Some simple nonlinear PDE's without solutions, Boll. Unione Mat. Ital. Sez. B Artic. Ric. Mat. (8) 1 (1998), 223-262. MR 99j:35001

[7] D. Del Santo, V. Georgiev and E. Mitidieri, Global existence of the solutions and formation of singularities for a class of hyperbolic systems, In: "Geometric Optics and Related Topics" (Eds. F. Colombini \& N. Lerner), Progress in Nonlinear Differential Equations and Their Applications, Vol. 32, pp. 117-140, Birkhäuser, Boston, 1997.

[8] K. Deng and H. A. Levine, The role of critical exponents in blow-up theorems: the sequel, J. Math. Anal. Appl. 243 (2000), 85-126. MR 2001b:35031

[9] V. A. Galaktionov and S. I. Pohozaev, Blow-up, critical exponents and asymptotic spectra for nonlinear hyperbolic equations: Math. Preprint Univ. of Bath 00/10, 2000.

[10] V. Georgiev, H. Lindblad and C. Sogge, Weighted Strichartz estimate and global existence for semilinear wave equation, Amer. J. Math. 119 (1997), 1291-1319. MR 99f:35134

[11] M. Guedda and M. Kirane, Criticality for some evolution equations, Differ. Uravn. 37 (2001), 610-622.

[12] F. John, Nonlinear wave equations, formation of singularities, University Lecture Ser. 2, Amer. Math. Soc., Providence, RI, 1990. MR 91g:35001

[13] V. A. Kondratiev and A. A. Kon'kov, On properties of solutions of a class of nonlinear second-order equations, Mat. Sb. 185 (1994), 81-94. MR 95i:35101

[14] V. V. Kurta, Certain problems of the qualitative theory of second-order nonlinear differential equations, Doctoral (Phys.-Math.) Dissertation, Moscow: Steklov Inst. Math., Russ. Acad. Sci., 1994.

[15] V. V. Kurta, On the absence of positive solutions to semilinear elliptic equations, Tr. Mat. Inst. Steklova 227 (1999), 162-169; English transl., Proc. Steklov Inst. Math. 1999, no. 4 (227), 155-162. MR 2001h:35062

[16] G. G. Laptev, Absence of global positive solutions for systems of semilinear elliptic inequalities in cone, Izv. Ross. Akad. Nauk Ser. Mat. 64 (2000), 107-124. 
[17] G. G. Laptev, On the absence of solutions to a class of singular semilinear differential inequalities, Tr. Mat. Inst. Steklova 232 (2001), 223-235.

[18] G.G. Laptev, Nonexistence of solutions to semilinear parabolic inequalities in cones, Mat. Sb. 192(10) (2001), 51-70.

[19] G.G. Laptev, Some nonexistence results for higher-order evolution inequalities in cone-like domains, Electron. Res. Announc. Amer. Math. Soc. 7 (2001), 87-93.

[20] H. A. Levine, The role of critical exponents in blow-up theorems, SIAM Rev. 32 (1990), 262-288. MR 91j:35135

[21] H.A. Levine and Q. Zhang, The critical Fujita number for a semilinear heat equation in exterior domains with homogeneous Neumann boundary values, Proc. Roy. Soc. Edinburgh A 130 (2000), 591-602. MR 2001g:35134

[22] E. Mitidieri and S.I. Pohozaev, Nonexistence of global positive solutions to quasilinear elliptic inequalities, Dokl. Russ. Acad. Sci. 57 (1998), 250-253.

[23] E. Mitidieri and S. I. Pohozaev, Absence of positive solutions for quasilinear elliptic problems on $\mathbf{R}^{N}$, Tr. Mat. Inst. Steklova 227 (1999), 192-222; English transl., Proc. Steklov Inst. Math. 1999, no. 4 (227), 186-216. MR 2001g:35082

[24] E. Mitidieri and S. I. Pohozaev, A Priori Estimates and Blow-up of Solutions to Nonlinear Partial Differential Equations and Inequalities, Nauka, Moscow, 2001 (Tr. Mat. Inst. Steklova 234).

[25] K. Mochizuki and R. Suzuki, Critical exponent and critical blow up for quasi-liner parabolic equations, Israel J. Math. 98 (1997), 141-156. MR 98b:35084

[26] R. G. Pinsky, Existence and nonexistence of global solutions for $u_{t}=\Delta u+a(x) u^{p}$ in $R^{d}$, J. Differential Equations 133 (1997), 152-177. MR 97k:35118

[27] S.I. Pohozaev, Essential nonlinear capacities induced by differential operators, Dokl. Russ. Acad. Sci. 357 (1997), 592-594.

[28] S. I. Pohozaev and A. Tesei, Blow-up of nonnegative solutions to quasilinear parabolic inequalities, Atti Accad. Naz. Lincei Cl. Sci. Fis. Mat. Natur. Rend. Lincei (9) Mat. Appl. 11 (2000), 99-109. MR 2001k:35306

[29] S. I. Pohozaev and A. Tesei, Instantaneous blow-up results for nonlinear parabolic and hyperbolic inequalities, Differ. Uravn. (to appear).

[30] A. A. Samarskii, V. A. Galaktionov, S. P. Kurdyumov and A.P. Mikhailov, Blow-up in quasilinear parabolic equations, Nauka, Moscow, 1987; English transl., Walter de Gruyter, Berlin/New York, 1995. MR 89a:35002 MR 96b:35003

[31] G. Todorova, B. Yordanov, Critical exponent for a nonlinear wave equation with damping, C. R. Acad. Sci. Paris, Ser. I 330 (2000), 557-562. MR 2001a:35025

[32] L. Veron and S. I. Pohozaev, Blow-up results for nonlinear hyperbolic inequalities, Ann. Scuola Norm. Sup. Pisa Cl. Sci. (4) 29 (2000), 393-420. MR 2001m:35227

[33] Qi Zhang, Blow-up results for nonlinear parabolic equations on manifolds, Duke Math. J. 97 (1999), 515-539. MR 2000d:35107

[34] Qi Zhang, A new critical behavior for nonlinear wave equations, J. Comput. Anal. Appl. 2 (2000), 277-292. MR 2001i:35212

[35] Qi Zhang, A blow-up result for a nonlinear wave equation with damping: The critical case, C. R. Acad. Sci. Paris, Série I 333 (2001), 109-114.

Department of Function Theory, Steklov Mathematical Institute, Gubkina str. 8, Moscow, Russia

E-mail address: laptev@home.tula.net 